\title{
A Xor THRESHOLd LOGIC IMPLEMENTATION THROUGH RESONANT TUNNELING DIODE
}

\author{
Nitesh Kumar Dixit ${ }^{1}$ and Vinod Kumari ${ }^{2}$ \\ ${ }^{1,2}$ Department of Electronics and Communication Engineering, BIET, Sikar \\ ${ }^{1}$ Nitesh20.dixit@gmail.com \\ ${ }^{2}$ vins.bhaskar16@gmail.com
}

\begin{abstract}
Resonant tunneling diodes (RTDs) have functional versatility and high speed switching capability. The integration of resonant tunneling diodes and MOS transistor makes threshold gates and logics. The design and fabrication of linear threshold gates will be presented based on a monostable bistable transition logic element. Each of its input terminals consist out of a resonant tunnelling diode merged with a transistor device. The circuit models of RTD and MOSFET are simulated in HSPICE. Two input XOR gate is designed and tested.
\end{abstract}

\section{Keywords}

MOSFET, RTD, SPICE, Threshold, TLG

\section{INTRODUCTION}

Energy quantization due to columbic repulsion of single charges and quantum confinement are their underlying physical phenomena to control the charge transport through this nanoscale structures. The use of quantum effects to reduce the logic depth of a circuit and a flexible and intuitive methodology to transfer give logic functionality into a circuit design [1]. The continuous scaling of CMOS has encouraged research into alternative structures for future logic devices. These devices are capable of high speed operation with reduced power consumption. RTDs operate on the principle of quantum mechanics. The tunneling of electrons through a potential barrier into quantized well states, and the result is resonances in the transmission characteristics. The RTD characteristic depends on quantum mechanics nature of the tunneling process [2].

The Resonant tunneling diode (RTD), have features of its low voltage operation, THz capabilities and negative differential resistance [2][3]. The Terahertz range is the RTD maximum operating frequency and offers a wide range of applications, in analog-digital converter (ADC), frequency divider or multiplier, oscillator [4]) as well as digital ("multi-value" logic [5] [6]) circuits. Its I-V characteristic presents an unusual negative differential resistance (NDR)[7]. Resonant Tunneling Diodes (RTDs) present very attractive characteristics, such as a high intrinsic cut-off frequency (theoretical value in the $\mathrm{THz}$ range) and current peaks associated with Negative Differential Resistance (NDR) regions.

\subsection{THRESHOLD LOGIC}

Threshold Logic (TL), as a powerful alternative to Boolean Logic (BL), was proposed in the late 1950 's as a result of the development of neural network theory, and was proven in theory to be

DOI : 10.5121/vlsic.2012.3511 
more efficient than Boolean Logic [8]. Recently, TL gates have been proposed in CMOS technology, e.g., Capacitive Threshold Logic [9], Differential Current-Switch Threshold Logic [10], and emerging nano-technologies, e.g., Single Electron Tunneling (SET) [11], Resonant Tunneling Diodes (RTD) [12], etc. While important steps have been made towards the hardware implementation of TL computing units the TL utilization in Very Large Scale Integration (VLSI) also requires the existence of high-level TL synthesis tools. The research has been done is very little on Threshold logic [13]. Recently, a synthesis tool has been proposed in [14], but it mostly treats Threshold Logic Gates (TLG) as conventional BL gates and the particularities of TL gates are almost completely ignored during the synthesis process.

\subsubsection{LINEAR THRESHOLD GATES}

The basic function of a linear threshold gate (LTG) is to differentiate between labelled points (vectors) belonging to two different classes [15] [16] [17]. An LTG maps a vector of input data, $\mathrm{x}$, into a single binary output $\mathrm{Y}$. The transfer function of an LTG is given analytically by the Input Output relation of threshold element is defined as

$$
\begin{aligned}
& V_{0}=X_{n} W_{n} \pm----\mp X_{2} W_{2}+X_{1} W_{1}+W_{0} \\
& V_{0}=X_{n} W_{n} \pm----\mp X_{2} W_{2}+X_{1} W_{1}-T \\
& V_{0}=\sum_{i=1}^{n} W_{i} X_{i}-T \Rightarrow \text { Weighted Sum of elements-Threshold }
\end{aligned}
$$

Where

$$
\begin{aligned}
& V_{0}=\sum_{i=1}^{n} W_{i} X_{i} \text { is weighted sum } \\
& Y=f\left(V_{0}\right) \text { where } \mathrm{f} \text { is function of Heaviside } \\
& \mathrm{Y}=1 \text { if } V_{0}>0 \text { or } \sum_{i=1}^{n} W_{i} X_{i}>\mathrm{T} \\
& \mathrm{Y}=0 \text { if } V_{0}<0 \text { or } \sum_{i=1}^{n} W_{i} X_{i}<\mathrm{T}
\end{aligned}
$$

Where

$$
\begin{aligned}
& \mathrm{X}_{\mathrm{i}}=\left[\mathrm{X}_{1} \mathrm{X}_{2^{------}} \mathrm{X}_{\mathrm{n}}\right] \\
& \mathrm{W}_{\mathrm{i}}=\left[\mathrm{W}_{1} \mathrm{~W}_{2}-----\mathrm{W}_{\mathrm{n}}\right] \text { are the inputs and weights respectively and }
\end{aligned}
$$

$\mathrm{T}$ is threshold constant.

The symbolic representation of an LTG with $n$ inputs is shown in Figure 1. A graphical representation of Equation 1 is shown in Figure 2. 


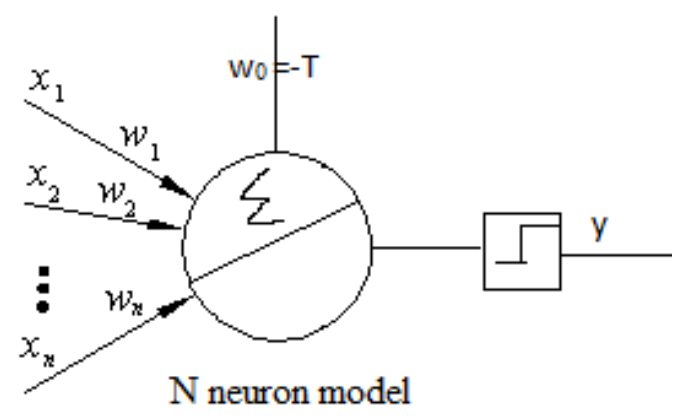

Figure 1 Symbolic representation of a linear threshold gate

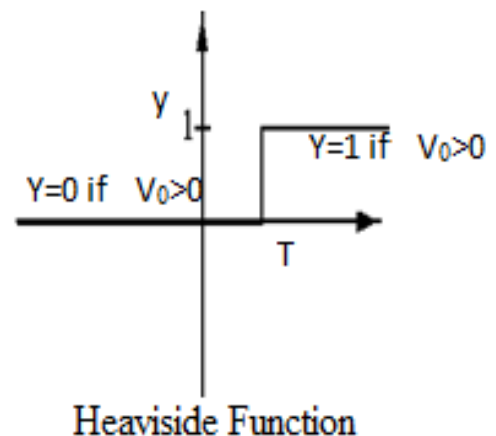

Figure 2 Transfer function.

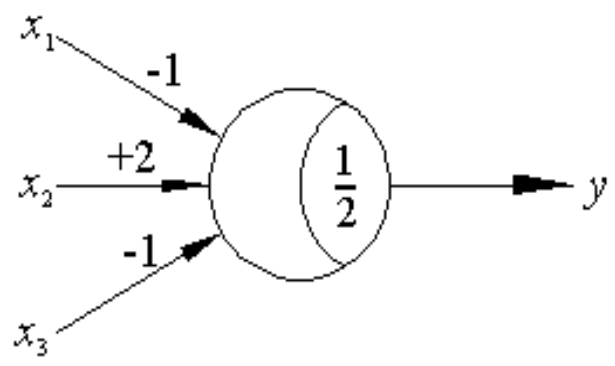

Figure 3 LTG realization of the Boolean function $y\left(x_{1}, x_{2}, x_{3}\right)=\overline{x_{1}} x_{2}+x_{2} \overline{x_{3}}$

Exclusive-OR (XOR) function $\left(x_{1}, x_{2}\right)=\overline{x_{1}} x_{2}+x_{1}, \bar{x}_{2}$ cannot be realized using a single LTG and is termed a threshold function Shown in Figure 3.

\subsubsection{GENERALIZED THRESHOLD GATES}

After MTTG (Multiple Threshold Threshold Logic Gate ), GTG is invented which reduce the complexity. GTG is Generalized Threshold Logic whose input implement according to positive and negative weight of threshold logic. We expect that the resulting Boolean function which is now only partially specified becomes a threshold function and hence realizable using a single 
LTG. The realization of a Boolean function by the above process leads to a generalized threshold gate (GTG). The general transfer characteristics for an $n$-input GTG are given by

$Y=1$ if $\sum_{i=1}^{n} W_{i} X_{i}, \sum_{i=0}^{n} \sum_{j=0}^{m} X_{i} Y_{j} W_{i j}>T$ and otherwise $Y=0$

\subsection{Resonant TunNELing Diode}

Tunneling diodes (TDs) have been used in widely applications like in achieving very high speed in wide-band devices and circuits that are beyond conventional transistor technology. The Resonant Tunneling Diode (RTD) is a particularly useful form of a tunneling diode, is the RTDs have been shown to achieve a maximum frequency of up to $2.2 \mathrm{THz}$ as opposed to $215 \mathrm{GHz}$ in conventional Complementary Metal Oxide Semiconductor (CMOS) transistors [18]. The very high switching speeds have allowed for a range of applications in wide-band secure communications systems and high-resolution radar and imaging systems for low visibility environments[19]

RTDs utilize a quantum well and with identically doped contacts to provide similar I-V characteristics. It consists of two heavily doped, narrow energy-gap materials encompassing an emitter region and a collector region. A quantum well in between two barriers of large band gap material, as shown in Figure 4 and the Energy Band Diagram of RTD shown in Figure 5. A Metal Organic Chemical Vapor Deposition using GaAs-AlGaAs is method of growth for this device. The barrier layers are around 1.5 to $5 \mathrm{~nm}$ thick and the quantum-well thickness is typically around $5 \mathrm{~nm}[20]$.

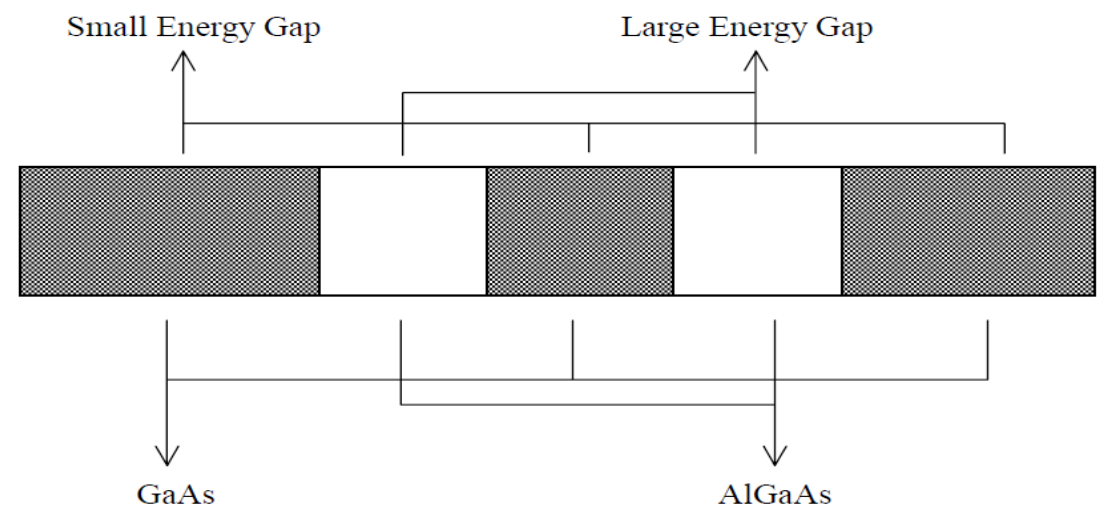

Figure 4. Structure of RTD (GaAs have small band gap and AlGaAs have large band gap). 


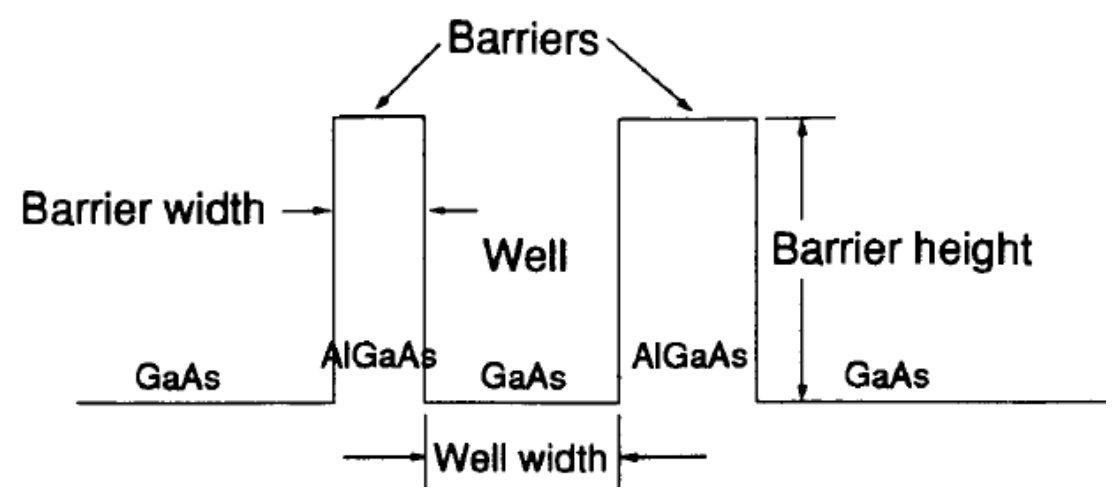

Figure 5. Energy Band Diagram of RTD

According to the graph on the emitter side, the current is at a maximum at the quasi-bound energy state indicated by the gray area in the quantum well shown in figure 4 and 5. RTD V-I characteristic shown in Figure 6.

\subsection{Previous WorK}

In threshold logic synthesis research was done mostly up to in the 1960s. In the determination of the input weights and threshold of a threshold function the approximation methods are used [21], [22]. Whether a function is threshold or not that is determine through admissible patterns on a Karnaugh map[23]. These methods are restricted to 10 or fewer variables due to computational complexity. Another methods like, the Linear programming and tabulation methods also have been used in [24] to determine if a function is threshold or not. The implementation of threshold gates using CMOS can be found in [25].

VLSI implementations survey report of threshold logic can be found in [26]. In [27], a branchand-bound algorithm is used to synthesize two-level threshold networks. Multi-level threshold network synthesis did not receive much attention in the 1950s and 1960s because efficient algorithms to factorize a multi-level network were unknown at that time. The one-to-one mapping is used by mostly methods, by replacing each Boolean gate in the network with a threshold gate. However, various algorithms exist today to compute the kernels and co-kernels of a network, they also used to perform algebraic or Boolean factorization [28].

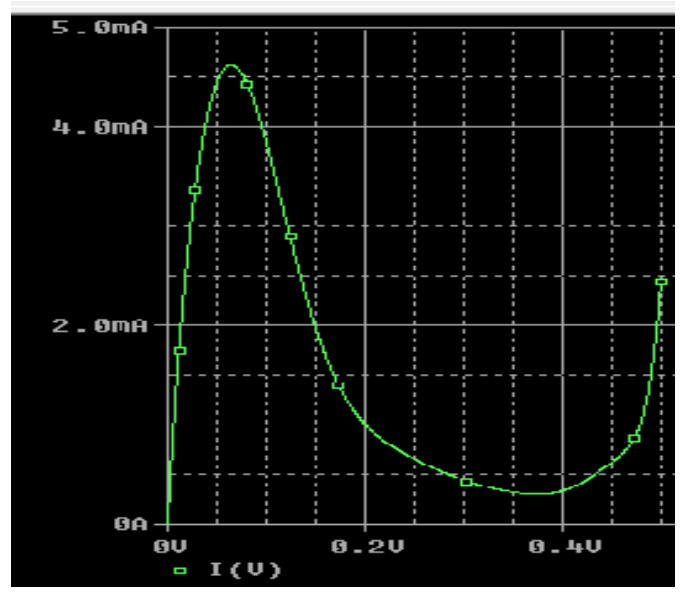

Figure 6. RTD V-I characteristic. 
International Journal of VLSI design \& Communication Systems (VLSICS) Vol.3, No.5, October 2012

\section{DESIGN AND IMPLEMENTATION}

\subsection{Methodology}

In this section, we present our test generation framework for threshold logic gate. The input is a Boolean Inputs and the output is a test set which the operation of XOR logic. The Boolean input is synthesized into a threshold logic gate where TLG contains weights corresponding to input. We then perform threshold logic using RTD simulation using HSpice.

RTD works on concept of MOBILE where MOBILE is monostable bistable logic element. MOBILE is series connection of two RTDs which contain load RTD and driver RTD. Whenever MOBILE have load RTD than positive weighted inputs are connect to parallel with load RTD and negative weighted inputs are connect parallel with driver RTD, the driver RTD and load RTD is works according to bias voltage (Vbias) and gives output as Vout. Equivalent circuit of XOR make using RTD and threshold logic and simulate it using HSpice. The RTD areas should be adjusted to appropriate values for correct operation, moreover, the transistors width may be tuned for better performance in the MOBILE-based circuits.

The New structure of XOR gate are designed. The new design input, and output values and margins, maximum operating frequency, delay of each transition, static and dynamic power dissipations of the new structure are calculated. The performance of new designed XOR gate is compared with other XOR gates. The results confirm that has a high performance of New designed structure. Designing new XORs is of much attention because they are the main part of an ALU and other digital devices.

The design of XOR gates of different structures were presented in literature over the years. Most of XOR gate circuits are based on FET transistors and also some XOR gates based on other logics. At some point, the voltage drop across the RTD will increase such that it is operating in the NDR region (i.e., the dynamic resistance will increase), and the input current will drop sharply, turning off the transistor, thus switching At some point, the voltage drop across the RTD will increase such that it is operating in the NDR region (i.e., the dynamic resistance will increase), and the input current will drop sharply, turning off the transistor, thus switching $\mathrm{V}_{\text {out }}$ back to $V_{\text {bias. }}$. Whenever we apply the bias voltage, current is increases at certain point, after that current is decreases at certain point and then current is increases, so that have two stable point and minimize the power dissipation than CMOS.

\subsection{XOR FUNCTION REPRESENTATION}

Here different implementation of XOR gate using the Threshold Logic and RTD/MOSFET. There is two threshold value so that XOR using MTTG is worked between

$$
T_{1} \prec \sum_{i=1}^{n} X_{i} W_{i} \prec T_{2}
$$

Here $\mathrm{W}_{1}=\mathrm{W}_{2}=1, \mathrm{~T}_{1}=1, \mathrm{~T}_{2}=2$ for XOR function.

Generalized Threshold Logic gate 1(GTG1), that is realized extended set of input variable as $\left(\mathrm{x}_{1}, \mathrm{x}_{2}, \mathrm{x}_{1} \& \mathrm{x}_{2}\right), \mathrm{W}_{1}=\mathrm{W}_{2}=1, \mathrm{~W}_{3}=-2, \mathrm{~T}=1$.

The XOR gate designed by the authors is illustrated in figure 7(a). It is an adaption of the MOBILE configuration with the use of both positive and negative weighted input sections. The XOR function is realized by placing a series connected MOSFET pair in parallel with the driver RTD. Using this configuration both inputs must be high to direct current away from the summing node ensuring that the circuit output is logic low when both inputs are high. In the XOR gate depletion type MOSFETs $(0.25 \mu \mathrm{m}$ gate length) are used above the summing node with 
enhancement mode devices $(0.25 \mu \mathrm{m}$ gate length) used in the series connected pair below the summing node. W:L ratios for all devices in both circuits are given in figure 4.

RTD areas given in figure 5 for both circuits are given in $2 \mu \mathrm{m}$ and the peak current density of the devices is $21 \mathrm{kA} / \mathrm{cm} 2$. Performance data for both circuits is given in table 1 . The threshold logic gate shown in Figure 7 (b) uses both positive and negative weighted inputs.

The gate is designed to output a logic high when $\mathrm{X} 1$ is high and $\mathrm{X} 2$ is low ( $\left.X 1 . X 2^{\prime}\right)$, and output logic low for all other input combinations. As with the XOR gate depletion mode MOSFETs $(0.25 \mu \mathrm{m}$ gate length) are used in the positive weighted section with enhancement mode MOSFETs $(0.25 \mu \mathrm{m}$ gate length) in the negative section.

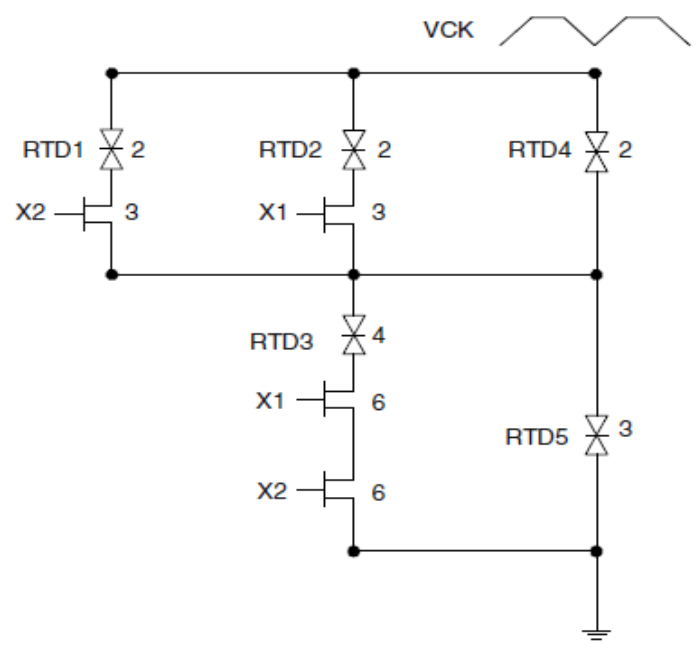

(a)

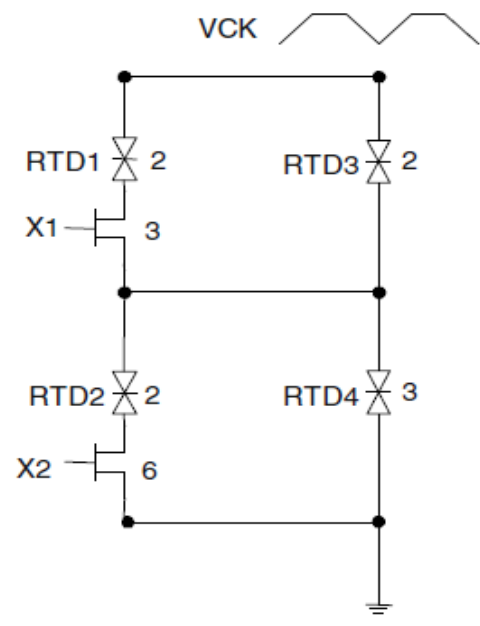

(b)

Figure 7. (a) Threshold logic XOR gate (b) Threshold logic gate for function X1.X2'

\section{RESULT}

The clock signal and $\mathrm{V}_{\text {bias }}$ is applied to the circuits and depict different sets of two inputs that are applied to the XOR gate and the HSPICE simulation results for these gates are depicted in Figure 8 and Figure 9.

Performance data for both circuits is given in table 1 . The gate is designed to output a logic low when $\mathrm{X} 1$ and $\mathrm{X} 2$ is high ( $X 1 . X 2)$, and output logic high for all other input combinations. As with the XOR gate depletion mode MOSFETs $(0.25 \mu \mathrm{m}$ gate length $)$ are used in the positive weighted section with enhancement mode MOSFETs $(0.25 \mu \mathrm{m}$ gate length) in the negative section.

Our proposed circuits are the first MOBILE based designs that implement two-input XOR circuits in a single structure, i.e. a single gate in transistor level. Implementing XOR function in single structure (single block) enables designers to design more efficient full adders in comparison with the previous full adders that are based on cascading of two two-input XOR gates [29][30][31] to achieve a three-input XOR gate. 
International Journal of VLSI design \& Communication Systems (VLSICS) Vol.3, No.5, October 2012

Table 1. Performance Data For the XOR gate and the $\mathrm{f}=\mathrm{X} 1$.X2'gate.

\begin{tabular}{|l|l|l|l|l|l|l|l|}
\hline Circuit & Clock & $\begin{array}{l}\text { Logic } \\
\text { High }\end{array}$ & $\begin{array}{l}\text { Logic } \\
\text { Low }\end{array}$ & $\begin{array}{l}\text { Rise } \\
\text { Time }\end{array}$ & Delay & Average & $\begin{array}{l}\text { Max } \\
\text { Power }\end{array}$ \\
\hline AND & $0.75 \mathrm{~V}$ & $0.701 \mathrm{~V}$ & $0.074 \mathrm{~V}$ & $150 \mathrm{pS}$ & $90 \mathrm{pS}$ & $167 \mu \mathrm{W}$ & $559 \mu \mathrm{W}$ \\
\hline Buffer & $0.75 \mathrm{~V}$ & $0.707 \mathrm{~V}$ & $0.0711 \mathrm{~V}$ & $160 \mathrm{pS}$ & $90 \mathrm{pS}$ & $95 \mu \mathrm{W}$ & $338 \mu \mathrm{W}$ \\
\hline XOR & $0.75 \mathrm{~V}$ & $0.707 \mathrm{~V}$ & $0.101 \mathrm{~V}$ & $160 \mathrm{pS}$ & $90 \mathrm{pS}$ & $114 \mu \mathrm{W}$ & $665 \mu \mathrm{W}$ \\
\hline X1.X2 & $0.75 \mathrm{~V}$ & $0.707 \mathrm{~V}$ & $0.071 \mathrm{~V}$ & $150 \mathrm{pS}$ & $90 \mathrm{pS}$ & $103 \mu \mathrm{W}$ & $343 \mu \mathrm{W}$ \\
\hline
\end{tabular}

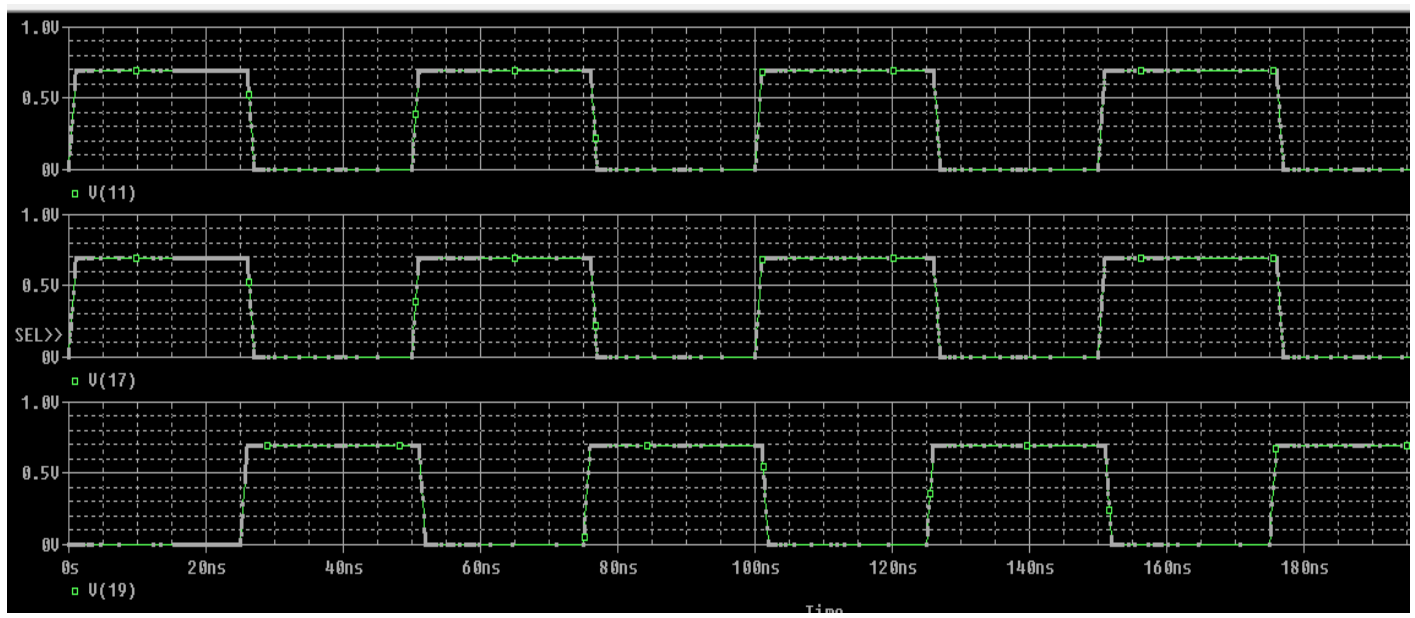

Figure 8. Voltage-Time Response of $X_{1} \overline{X_{2}}$ function $(v(17), v(19)$ are input $\& v(11)$ is output)

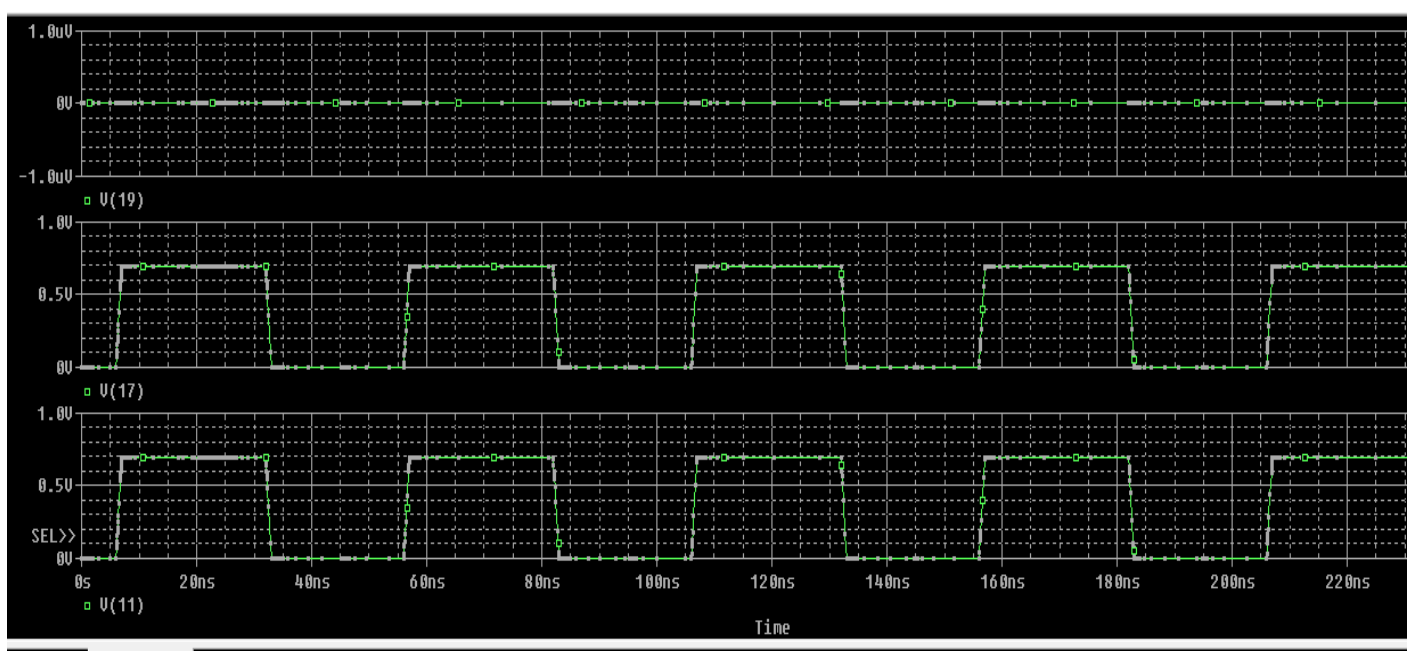

Figure 9.Voltage -Time Response of XOR function (v(17),v(19) are input \& v(11) is output)

\section{Conclusion}

In this paper, to the best of authors' knowledge, a new two-input XOR gate circuit and a new two-input XOR gate circuit based on generalized threshold gate (GTG) are introduced for the first time. A simulation for all combination of inputs is done and the results are drawn. RTD designs can offer a reduction in component count by up to $40 \%$ when compared with the equivalent 
CMOS logic family. Due to the threshold logic design style, which enables an increased low-level parallelism of computing multiple input signals, the advantage of the RTD circuits increases when equivalent circuit areas are compared. The main reason is that the threshold logic design eliminates the series connections of transistors in XOR-like gate configurations. Due to long series connections of p-MOSFETs in CMOS devices result in a large area consumption increases.

\section{ACKNOWLEDGMENT}

The authors would like to thank Dr. M.J. Siddiqui, Mrs. Emrati Bhaskar regarding HSPICE RTD model.

\section{REFERENCES}

[1] "Semiconductor Industries Association Roadmap." http://public.itrs.net.

[2] Madhukar Reddy, Schottky-collector Resonant Tunnel Diodes for Sub-Millimeter-Wave Applications http://www.ece.ucsb.edu/Faculty/rodwell/Downloads/theses/madhu_thesis.pdf (1997)

[3] M. Reddy, S. C. Martin, "Monolithic Schottky-Collector Resonant Tunnel Diode Oscillator Arrays to 650 GHz," IEEE Electron Device Letters 18, 218-221.

[4]. P. Sun, G. Haddad, "Resonant Tunneling Diodes: Models and Properties," IEEE 86, 641-660 (1998).

[5] T. Akeyoshi, H. Matsuzaki, T. Itoh, T. Waho, J. Osaka and M. Yamamoto, "Applications of Resonant-Tunneling Diodes to High-Speed Digital ICs,” Indium Phosphide and Related Materials 11, 405-410 (1999)

[6] M. J. Avedillo, J. M. Quintana and H. Pettenghi, "Logic Models Supporting the Design of MOBILEbased RTD Circuits," Architecture and Processors (ASAP'05), 2005.

[7] K. S. Berezowski, "Compact Binary Logic Circuits Design Using Negative Differential Resistance Devices," Electronics Letters, Vol. 42, No. 16, 3rd August, 2006.

[8] P. M. Lewis II and C. L. Coates. Threshold Logic. New York: Wiley, 1967.

[9] Hakan Ozdemir, Asim Kepkep, Banu Pamir, Yusuf Leblebici, and Ugur Cilingiroglu. "A Capacitive Threshold-Logic Gate". IEEE Transaction On Computer-Aided Design Of Integrated Circuits And Systems, 31(8):1141-1149, August 1996.

[10] M. D. Padure, S. D. Cotofana, and S. Vassiliadis. Cmos implementation of generalized threshold functions. In Proceedings of the International Work-conference on Artificial and Natural Neural Networks (IWANN2003), pages 65-72, June 2003.

[11] C. R. Lageweg, S. D. Cotofana, and S. Vassiliadis. A full adder implementation using set based linear threshold gates. In Proceedings 9th IEEE International conference on electronics, circuits and systems - ICECS 2002, pages 665-669, September 2002.

[12] K. Maezawa, H. Matsuzaki, M. Yamamoto, and T Otsuji. " Highspeed and low-power operation of a resonant tunneling logic gate mobile". In IEEE Electron Device Letters, pages 80-82, March 1998.

[13] Michael Dertouzos. Threshold Logic: A Synthesis Approach. M.I.T. Press, 1965.

[14] H. Pettenghi, M. J. Avedillo, J. M. Quintan, "Using Multi-Threshold Threshold Gates in RTD-Based Logic Design,” Elsevier, Microelectronics Journal, Vol. 39, pp. 241-247, 2008.

[15] Rui Zhang, Pallav Gupta, Lin Zhong, and Niraj K. Jha. "Threshold Network Synthesis and Optimization and Its Application to Nanotechnologies" . IEEE Transaction On Computer-Aided Design Of Integrated Circuits And Systems, 24(1):107-118, January 2005.

[16] G. De Micheli. Synthesis and Optimization of Digital Circuits. McGraw Hill, 1993.

[17] S. Muroga. Threshold Logic and Its Application. Wiley and Sons Inc., 1971

[18] R. Kosik, Resonant Tunneling Diode Structure http://www.iue.tuwien.ac.at/phd/kosik/node64.html

[19] M. J. Avedillo, J. M. Quintan , H. Pettenghi, "Increased Logic Functionality of Clocked SeriesConnected RTDs," IEEE Transactions on Nanotechnology, Vol. 5, No. 5, Sept. 2006.

[20] G. Witt, Resonant Tunneling Diode Research, http://www.afrlhorizons.com/Briefs/0006/OSR0001.html

[21] R. O. Winder, Threshold logic, Ph.D. dissertation, Princeton University, 1962.

[22] M. L. Dertouzos, Threshold Logic: A Synthesis Approach. Cambridge, MA: The M.I.T. Press, 1965.

[23] Z. Kohavi, Switching and Finite Automata Theory. New York, NY: McGraw-Hill, 1978

[24] S. Muroga, Threshold Logic and its Applications. New York, NY: John Wiley, 1971. 
International Journal of VLSI design \& Communication Systems (VLSICS) Vol.3, No.5, October 2012

[25] G. E. Sobelman and K. Fant, "CMOS circuit design of threshold gates with hysteresis," in Proc. Inte. Conf. of Circuits \& Systems, vol. 2, May 1998, pp. 61-64.

[26] V. Beiu, J. M. Quintana, and M. J. Avedillo, "VLSI implementations of threshold logic - A comprehensive survey," Tutorial at International Joint Conference of Neural Networks, July 2003.

[27] A. L. Oliveira and A. Sangiovanni-Vincentelli, "LSAT - An algorithm for the synthesis of two level threshold gate networks," in Proceeding International Joint Conference of. Computer-Aided Design, Nov. 1991, pp. 130-133.

[28] G. D. Hachtel and F. Somenzi, "Logic Synthesis and Verification Algorithms". Norwell, MA: Kluwer Academic Publishers, 1998.

[29] E. M. Sentovich et al., "Sequential circuit design using synthesis and optimization", in Proc. Int. Conf. Computer Design, Oct. 1992, pp. 328-333.

[30] Krzysztof Kozminski. "Benchmarks for layout synthesis evolution and current status". In 28th ACM/IEEE Design Automation Conference, pages 255-270, 1991.

[31] Rui Zhang, Pallav Gupta, Lin Zhong, and Niraj K. Jha. Threshold Network Synthesis and Optimization and Its Application to Nanotechnologies . IEEE Transaction On Computer-Aided Design Of Integrated Circuits And Systems, 24(1):107-118, January 2005.

\section{Authors}

The First author is Nitesh Kumar Dixit. He is currently working as Asst. Prof., in BIET, Sikar; He has Completed his M.Tech degree in Embedded System, SRM University, Chennai and B.E.(ECE) SEC,Dundlod (jhujhunu). His area of interests is VLSI, Digital Circuits, Embedded System, BIST, SOC etc.

The Second author is Ms. Vinod Kumari. She is currently working as Asst. Prof., in BIET, Sikar; She has obtained her M.Tech (VLSI Design) degree from Banasthali University, Rajasthan and B.E.(CSE) from SEC,Dundlod (Jhujhunu). Her area of interests is VLSI, Digital Circuits, Embedded System etc.
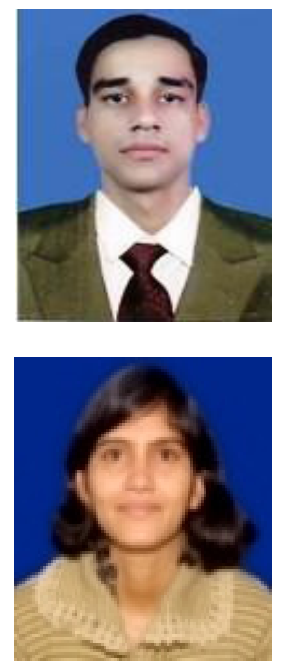